\title{
EXPONENTIAL ASYMPTOTICS WITH A SMALL EXPONENT
}

\author{
$\mathrm{BY}$ \\ G. C. KEMBER (Dept. of Engineering Mathematics, DalTech, Dalhousie University, Halifax, \\ Nova Scotia, Canada), \\ A. C. FOWLER (Mathematical Institute, Oxford University, 24-29 St. Giles', Oxford, UK), \\ J. D. EVANS (Dept. of Mathematics, University of Wales, Aberystwyth, Ceredigion, UK),
}

AND

S. B. G. O'BRIEN (Dept. of Mathematics, University of Limerick, Republic of Ireland)

\begin{abstract}
Analytic and numerical solutions are considered to a simple model problem which contains a surprisingly complicated solution structure. Asymptotic solutions are sought when a parameter that appears as an exponent in the independent variable is small, the solution then exhibiting a sudden change in slope over a region that is exponentially thin. A straightforward approach using matched asymptotic expansions immediately reveals inadequacies of this method due to the requirement of an outer solution that needs to be evaluated beyond all orders in order to match to a suitable inner solution. This behaviour is elucidated by studying first the asymptotic structure of the solution using an exact integral, which explicitly reveals the need for the inclusion of exponentially small terms in the expansions. It is then shown how a direct asymptotic solution of the differential equation can be obtained by using Borel summation to evaluate the outer solution to exponential accuracy. Further, as a practical alternative, it is shown how these exponentially improved approximations can be made when an exact numerical solution is available and without recourse to the general term of the outer or inner expansions.
\end{abstract}

1. Introduction. Exponential asymptotics, or asymptotics beyond all orders, is a subject that has been very much in vogue since Berry's development [1] of a uniform asymptotic expansion to describe Stokes's phenomenon. Berry built on earlier work by Dingle [2], in which the concept of exponentially improved asymptotic expansions was introduced. For an asymptotic expansion in powers of a small parameter $\varepsilon$, the number of terms $N(\varepsilon)$ is selected so that the first neglected term is minimal. The resulting error in the expansion is typically exponentially small in $\varepsilon$. By evaluating the exponentially small error as a function of the phase, Berry was able to compute universal error function

Received July 13, 1998.

2000 Mathematics Subject Classification. Primary 34E18, 34E20, 34M30. 
transitions in the asymptotic expansions of integrals exhibiting Stokes's phenomenon, such as that defining the Airy function.

Integrals of this type can also be defined as solutions of differential equations, and it is an obvious question to ask whether it is possible to construct exponentially accurate solutions directly from the differential equation. Reference [9] used matched asymptotic expansions for differential equations to construct transition profiles as described above for certain integrals. A more direct approach to a problem of this type is the pioneering work by Kruskal and Segur [5], who derived exponentially small corrections in the solution of a nonlinear problem by embedding the equation with a complex time variable, and then finding correct inner and outer expansions near and far from a singularity of the equation. However, their approach is unable to describe Berry's delicate switching behaviour.

The problem we wish to consider here has certain features in common with these other problems, but it is sufficiently novel that we have had to develop a different procedure to deal with the solution. The purpose of our pedagogical presentation is to illustrate the idea that these same procedures may have wide applicability in other similar problems. In particular, we find that satisfactory matched asymptotic expansions for the solution require one of the asymptotic expansions to be prescribed to all algebraic orders, and in general this requires the use of Borel resummation (applied to sequences of operators) to provide an exponentially improved series. This can be done straightforwardly as long as the equation is quasi-linear, and the linear differential part of the equation has constant coefficients. This restriction is no more serious than that in the majority of the examples that have been studied in the literature. A review of the development of these recent ideas is given in [10], and an indication of the different applications where exponentially small terms occur is in the book [11].

The example problem we consider is

$$
\frac{d y}{d x}=\frac{1}{y^{\varepsilon}}-2, \quad y\left(x_{0}\right)=y_{0},
$$

where $y_{0}>2^{-1 / \varepsilon}$ and $x_{0}<0$ are the ranges of interest for the initial condition, and $\varepsilon$ is a small, positive parameter. The solution is analyzed for $x \geq x_{0}$, wherein it is monotonic decreasing. This model problem is derived in [3] as the canonical form of a set of equations describing the physical problem of surging ice sheets (see also [4] and $[8])$.

The exact solution to (1) can be written implicitly as

$$
x=x_{0}+\int_{y}^{y_{0}} \frac{u^{\varepsilon}}{2 u^{\varepsilon}-1} d u,
$$

and it is the asymptotic structure of this solution in the limit $\varepsilon \rightarrow 0^{+}$that will be of interest. For this limit, the solution exhibits a sharp transition region, shown in Fig. 1, separating the initial dominant behaviour in $x=O(1)$, where $y \sim\left(y_{0}+x_{0}-x\right)$, and that for $x>y_{0}+x_{0}$, where $y \sim 2^{-1 / \varepsilon}$. The aim here will be to show the necessity for an asymptotic expansion capable of representing the above solution to exponential accuracy, particularly through the sharp transition region, and subsequently we will show how such an expansion can be generated directly from the differential equation. 


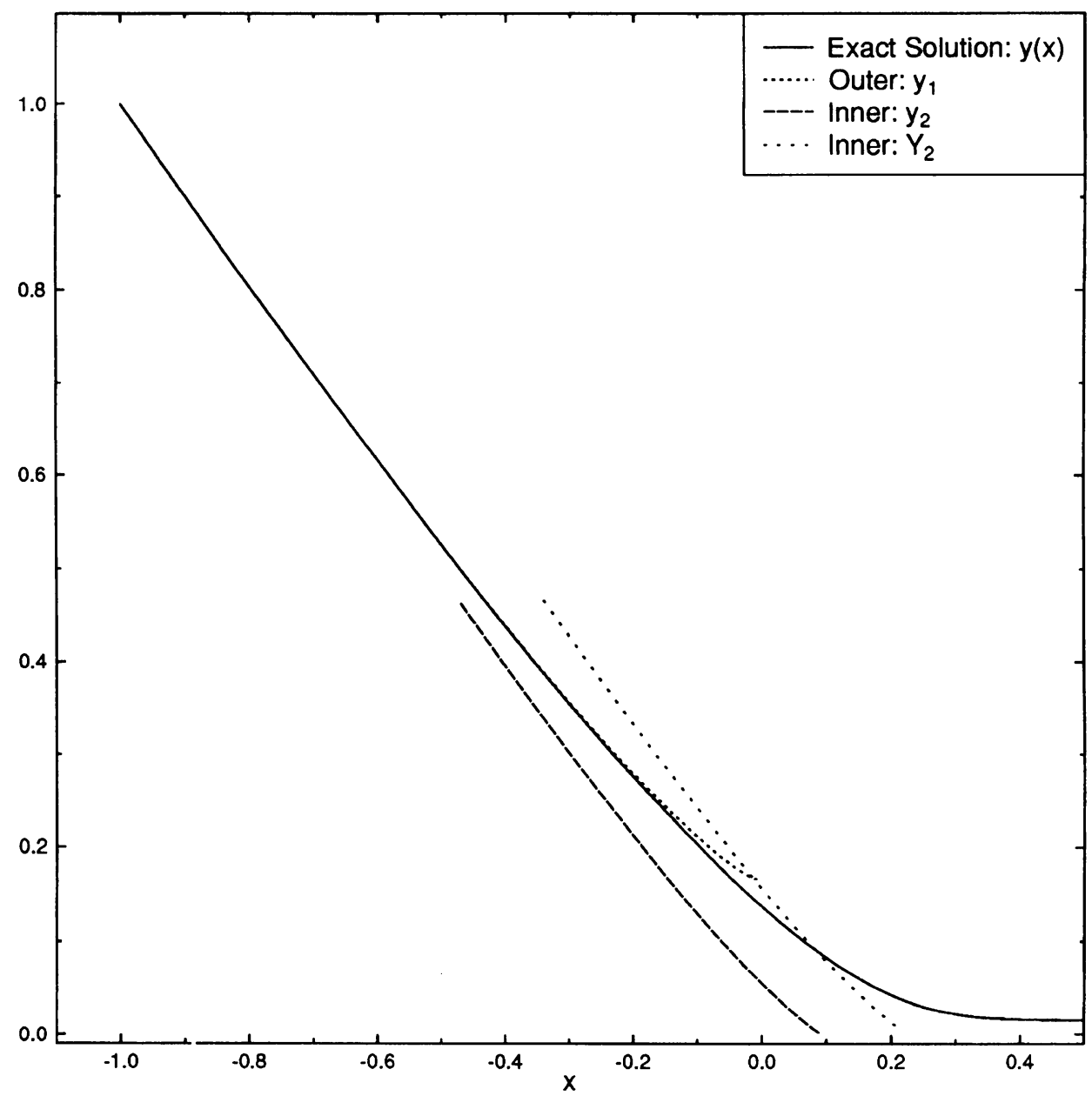

FIG. 1. Illustration of the numerical solution of (1), together with the two-term expansions of $y_{1}(x)$ and $y_{2}(\xi)$, depicted for $\varepsilon=1 / 6$ and the initial condition $x_{0}=-1, y_{0}=1$. The sharp transition region is apparent at $x=O(\varepsilon)$. The error made by $y_{2}(\xi)$ with a second-order approximation to the shift $A(1 / 6)=1 / 6$ is substantial. Also shown is the two-term expansion of $y_{2}(\xi)$, but with the shift $A(1 / 6) \approx 0.3$ found numerically, and $y_{2}(\xi)$ has been relabelled as $Y_{2}(\xi)$ for clarity. The improvement in $Y_{2}(\xi)$ over $y_{2}(\xi)$ is clear.

The difficulties inherent in providing a matched asymptotic expansion for this problem should be understood first, and are most easily illustrated by showing why a straightforward approach is inadequate. An outer expansion in regular powers of $\varepsilon$ that satisfies the initial condition is

$$
\left.y_{1}(x)=\left(y_{0}+x_{0}-x\right)+\varepsilon_{(}\left(y_{0}+x_{0}-x\right) \ln \left(y_{0}+x_{0}-x\right)-y_{0} \ln y_{0}+x-x_{0}\right)+O\left(\varepsilon^{2}\right) .
$$


The expansion for $y_{1}(x)$ is invalid when $\varepsilon \ln \left(y_{0}+x_{0}-x\right)=O(1)$, and an inner region $\xi=O(1)$ is introduced with the scalings

$$
\xi=-\varepsilon \ln [A(\varepsilon)-x], \quad y_{2}=\exp (\xi / \varepsilon) y,
$$

where the function $A(\varepsilon)$ is a shift required to enable matching with the outer solution. The expansion of $y_{2}(\xi)$ is given by

$$
y_{2}(\xi)=\left(2-e^{\xi}\right)+\varepsilon e^{\xi}\left[-1+\ln \left(2-e^{\xi}\right)\right]+O\left(\varepsilon^{2}\right),
$$

and the shift $A(\varepsilon)$ correct to second order is

$$
A(\varepsilon)=\left(y_{0}+x_{0}\right)+\varepsilon\left(y_{0}-y_{0} \ln y_{0}\right)+O\left(\varepsilon^{2}\right) .
$$

In terms of the outer variables, the inner region is exponentially thin and, moreover, the solution is exponentially small. Thus, the first sign of difficulty arises as the shift $A(\varepsilon)$ needs to be exponentially accurate, if it is to be of practical use. Following [2], this requires calculation of the general term in the expansion for $A(\varepsilon)$, and then the resumming of the divergent tail of the expansion beyond its minimum term, to yield the leading-order exponentially small correction term.

To examine the effect of the shift $A(\varepsilon)$, the numerical solution of (1), with $x_{0}=$ $-1, y_{0}=1$, and $\varepsilon=1 / 6$, is depicted in Fig. 1 along with the two-term expansions of $y_{1}(x)$ and $y_{2}(\xi)$. Although $y_{2}(\xi)$ and $A(\varepsilon)$ are specified to second order, the error made by $y_{2}(\xi)$ in Fig. 1 is substantial. This is most noticeable in the inner solution, which is clearly shifted from the exact solution. Numerical calculation of $A(\varepsilon)$, using (55), shows that in fact $A(1 / 6) \approx 0.3$. This point is further illustrated in Fig. 1, where the twoterm expansion of $y_{2}(\xi)$ is depicted, where $A(1 / 6) \approx 0.3$ is used in (4) (rather than its value of $1 / 6$ given by $(6)$ ), and this shifted inner expansion is denoted by $Y_{2}(\xi)$ to avoid confusion. The improvement in $Y_{2}(\xi)$ over $y_{2}(\xi)$ is plain. Nevertheless, the error made by $Y_{2}(\xi)$ is still significant, and this is particularly evident within the region of overlap between $Y_{2}(\xi)$ and $y_{1}(x)$, for negative values of $x$ of $O(\varepsilon)$.

The inner expansion (5) breaks down as $\xi \rightarrow \ln 2$ and this suggests a third region $X=O(1)$ with the scalings

$$
X=\varepsilon 2^{1 / \varepsilon}(A(\varepsilon)-x), \quad y_{3}=2^{1 / \varepsilon} y .
$$

The following expansion is obtained:

$$
y_{3}(X)=Y_{0}(X)+\varepsilon\left(c_{1}-\frac{Y_{0}(X)}{2}\right) \ln Y_{0}(X)+O\left(\varepsilon^{2}\right),
$$

where $Y_{0}(X)$ satisfies

$$
2 X=\int_{\ln Y_{0}(X)}^{c_{0}} w^{-1} e^{w} d w
$$

and the constants $c_{0}, c_{1}$ are to be determined by matching with the inner solution (5). At this point, further difficulty arises as the full inner expansion of the series (5) is required to match to the first term in (8) as discussed in [3]. Consequently, the constant $c_{0}$, which represents an origin shift for the function $Y_{0}(X)$, cannot be determined by matching to the algebraic inner expansion (5). 
For algebraic convenience, and ease of discussion in subsequent sections, the change of variables

$$
\phi=\frac{x}{y}, \quad z=y^{-\varepsilon}
$$

is introduced, so that (1) becomes

$$
\phi-\varepsilon z \frac{d \phi}{d z}=\frac{1}{z-2} \quad \text { with } \phi\left(z_{0}\right)=\phi_{0},
$$

where $z_{0}=y_{0}^{-\varepsilon}, \phi_{0}=x_{0} / y_{0}$, and $0<z_{0} \leq z<2$. This form is linear in $\phi(z)$, and the exact solution (2) can be written as

$$
\phi(z)=\phi_{0}\left(\frac{z}{z_{0}}\right)^{1 / \varepsilon}+z^{1 / \varepsilon} \int_{z_{0}}^{z} \frac{d\left(w^{-1 / \varepsilon}\right)}{(w-2)}, \quad 0<z_{0} \leq z<2 .
$$

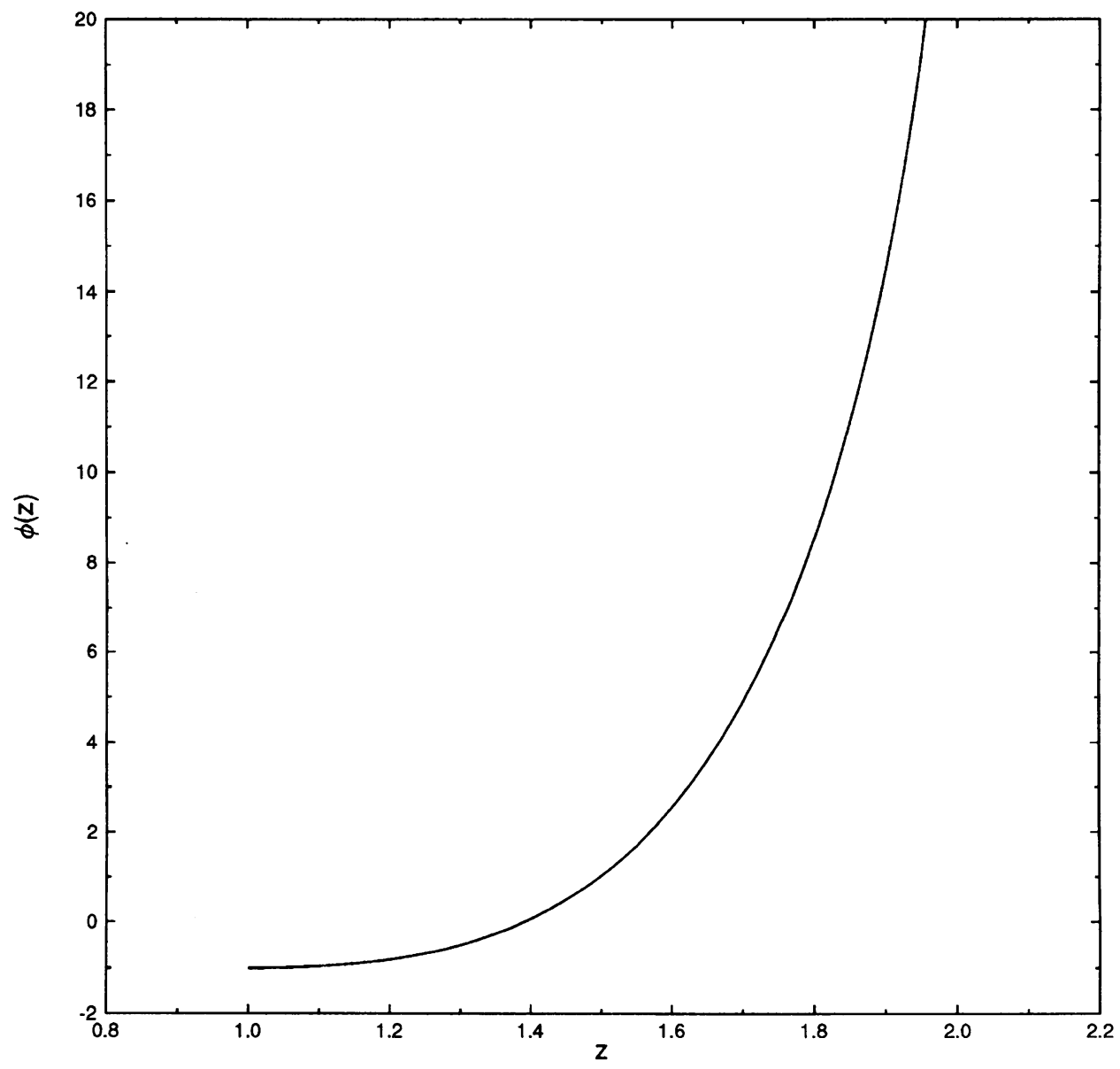

FIG. 2. The numerical solution of the transformed problem in (11) for $\phi(z)$ is depicted using the same parameters and condition as in Fig. 1 , i.e., $\varepsilon=1 / 6$ with $z_{0}=1, \phi_{0}=-1$. 
To fix ideas, the numerical solution in Fig. 1 is shown in Fig. 2 in terms of the transformed variables $\phi$ and $z$. The sharp transition is now near $z=2$.

The asymptotic structure of the solution in terms of $\phi$ and $z$ has been given, to leading order, in [3], but they were unable to compute the exponentially small corrective terms which, as we have intimated above, are crucial for a practically useful approximation. Reference [8] studied the structure of the solution using exact series solutions (cf. (15) below), which truncate when $\varepsilon=1 / n$, where $n$ is an integer. In order for us to reclaim the exponentially small terms, we proceed in the following section to compute the asymptotic structure by using resummation techniques applied to the exact integral form of the solution. We then attempt, in Sec. 3, to recover this form of the solution directly from the differential equation.

2. Exponentially small corrections to Laplace integrals. We obtain asymptotic approximations to (12) using Laplace's method. A key observation in the application of Watson's lemma to Laplace-type integrals is that exponentially small remainder terms typically arise through both the resumming technique alluded to earlier, and also the constant finite limits of integration. The latter arise in (12) from the variable limit $z$ and the constant limit $z_{0}$. To facilitate their calculation, the solution (12) for $\phi(z)$ is equivalently written as

$$
\phi(z)=\left(\frac{z}{z_{0}}\right)^{1 / \varepsilon}\left[\phi_{0}-I\left(z_{0}\right)\right]+I(z),
$$

where $I(z)$ denotes the Cauchy principal value integral

$$
I(z)=-z^{1 / \varepsilon} f_{z}^{\infty} \frac{d\left(w^{-1 / \varepsilon}\right)}{w-2} .
$$

In what follows, it is assumed that $z_{0}$ and $\phi_{0}$ are $O(1)$, and this will be seen to be without loss of generality. To derive an asymptotic expansion of $I(z)$, for small, positive $\varepsilon$, (14) is repeatedly integrated by parts, to give

$$
I(z) \sim \frac{1}{z-2}+\sum_{n=1}^{\infty} \frac{\varepsilon^{n} n !}{\Pi_{j=1}^{n}(j \varepsilon-1)} \frac{z^{n}}{(z-2)^{n+1}}, \quad \text { as } \varepsilon \rightarrow 0 .
$$

The series in (15) is left in this useful form, but in principle (15) would be further expanded for small, positive $\varepsilon$ and the latter is the form encountered when proceeding from the differential equation in Sec. 3 .

To develop an exponentially accurate approximation to $I(z),(15)$ is truncated after $N+1$ terms, and written as

$$
I(z)=F(z)+S_{N}(z)
$$

where

$$
F(z)=\sum_{n=0}^{N} \frac{\varepsilon^{n} n !}{\Pi_{j=1}^{n}(j \varepsilon-1)} \frac{z^{n}}{(z-2)^{n+1}}
$$


is a Poincare series and $S_{N}(z)$ is the remainder, which may be expressed in the integral form

$$
S_{N}(z)=-z^{1 / \varepsilon} \frac{\varepsilon^{N}(N+1) !}{\Pi_{j=1}^{N}(j \varepsilon-1)} \int_{z}^{\infty} \frac{w^{-\frac{1}{\varepsilon}+N} d w}{(w-2)^{N+2}} .
$$

Interest lies in the form of the leading-order approximation to $S_{N}(z)$, for small, positive $\varepsilon$, which we obtain by applying the procedure enunciated in [9]. Using this convenient integral form, the remainder satisfies

$$
S_{N}(z)-\varepsilon z \frac{d S_{N}(z)}{d z}=\frac{-\varepsilon^{N+1}(N+1) !}{\Pi_{j=1}^{N}(j \varepsilon-1)} \frac{z^{N+1}}{(z-2)^{N+2}},
$$

the right-hand side of which can be shown to be minimal at

$$
N=\frac{1}{\varepsilon}\left(1-\frac{z}{2}+\frac{\varepsilon}{2}(1-z)+O\left(\varepsilon^{2}\right)\right)
$$

Using this value of $N$ in the right-hand side of (19), and expanding for small, positive $\varepsilon$, gives to first order

$$
S_{N}(z)-\varepsilon z \frac{d S_{N}(z)}{d z}=-\sqrt{\frac{\pi}{2 \varepsilon}} \sqrt{\frac{z}{2-z}}\left(\frac{z}{2}\right)^{1 / \varepsilon} .
$$

Integrating (21), a first-order approximation to the remainder is

$$
S_{N}(z)=[G(z, \varepsilon)+H(\varepsilon)]\left(\frac{z}{2}\right)^{1 / \varepsilon},
$$

where

$$
G(z, \varepsilon)=\frac{1}{\varepsilon^{3 / 2}} \sqrt{\frac{\pi}{2}}\left[\sin ^{-1}(z-1)\right]
$$

and $H(\varepsilon)=O\left(\varepsilon^{-3 / 2}\right)$ since by choice, $S_{N}$ is the minimal term in the expansion (17). The source of the exponentially small correction $H(\varepsilon)(z / 2)^{1 / \varepsilon}$ is the rewriting of the quadrature in (12) in terms of two integrals, both with upper limits at infinity. This procedure then shows that

$$
I(z) \sim F(z)+[G(z, \varepsilon)+H(\varepsilon)]\left(\frac{z}{2}\right)^{1 / \varepsilon} \quad \text { as } \varepsilon \rightarrow 0 .
$$

Using (24) in (13) gives the expression

$$
\phi(z) \sim\left[\phi_{0}-F\left(z_{0}\right)\right]\left(\frac{z}{z_{0}}\right)^{1 / \varepsilon}+\left[G(z, \varepsilon)-G\left(z_{0}, \varepsilon\right)\right]\left(\frac{z}{2}\right)^{1 / \varepsilon}+F(z),
$$

where it is noted that the term $H(\varepsilon)$ does not appear through cancellation. Further, this last expression indicates the presence of three gauges: transcendentally large terms of order $\left(z / z_{0}\right)^{1 / \varepsilon}$, algebraic terms of order $\varepsilon^{n}$, and transcendentally small terms of order $(z / 2)^{1 / \varepsilon}$.

Clearly, (25) becomes invalid near $z=2$ and an inner region is required. To obtain an expansion of $\phi(z)$ valid near $z=2$, the transformation $u=(z / 2)^{1 / \varepsilon}$ is introduced 
together with the definitions $J(u)=I\left(2 u^{\varepsilon}\right)$ and $\phi(u)=\phi\left(2 u^{\varepsilon}\right)$ so that (13) may be written as

$$
\Phi(u)=u\left(\frac{2}{z_{0}}\right)^{1 / \varepsilon}\left[\phi_{0}-I\left(z_{0}\right)\right]+J(u)
$$

where

$$
J(u)=\frac{u}{2} f_{\ln u}^{\infty} \frac{e^{-r}}{e^{\varepsilon r}-1} d r .
$$

We evaluate an asymptotic approximation for $J(u)$ using Laplace's method. Expanding the integrand for $\varepsilon \ll 1$, we obtain

$$
J(u) \sim \frac{u}{2 \varepsilon} f_{\ln u}^{\infty} \frac{e^{-r}}{r} d r-\frac{1}{4}+\frac{1}{4} \sum_{n=1}^{\infty} \sum_{m=0}^{2 n-1} \frac{B_{2 n} \varepsilon^{2 n-1}(\ln u)^{m}}{n m !},
$$

where $B_{2 n}$ are the Bernoulli numbers. Thus, near $z=2$, we have

$$
\begin{aligned}
\Phi(u) \sim\left[\left(\frac{2}{z_{0}}\right)^{1 / \varepsilon}\left[\phi_{0}-F\left(z_{0}\right)\right]-G\left(z_{0}, \varepsilon\right)-H(\varepsilon)\right] u \\
+\frac{u}{2 \varepsilon} f_{\ln u}^{\infty} \frac{e^{-r}}{r} d r-\frac{1}{4}+\frac{1}{4} \sum_{n=1}^{\infty} \sum_{m=0}^{2 n-1} \frac{B_{2 n} \varepsilon^{2 n-1}(\ln u)^{m}}{n m !} .
\end{aligned}
$$

The unknown constant, $H(\varepsilon)$, does not cancel in this formula for $\Phi(u)$ and must be evaluated. We might suppose that, in analogy to the procedure we followed in approximating the outer solution (25), a resummation of the asymptotic series in (29) would yield a cancelling term, but a little thought shows that this is not the case. In fact, if the upper limit in (27) is replaced by $L / \varepsilon$, where $L$ is $O(1)$, then the asymptotic expansion (28) is unaltered; the implication is that the series itself cannot be used in this way. This failure is especially significant since it occurs in spite of our knowledge of the general term in both inner and outer expansions. Therefore, we wish to develop a procedure to evaluate "exponentially accurate" approximations to the solution in both inner and outer regions. The ability to do this requires sacrifice. First, we provide a methodology using Borel summation, which (unfortunately) requires a formal (if not explicit) expression for the general term. Second, we suggest a method which works directly from the differential equation, and which does not require the general term: the cost here is that matching of the inner and outer expansions must be done using an exact numerical solution of the equation.

3. Matched asymptotic expansions. Considering the differential equation in the form (11), an expansion for small, positive $\varepsilon$ leads to the outer expansion

$$
\phi(z)=\frac{1}{z-2}-\frac{\varepsilon z}{(z-2)^{2}}+O\left(\varepsilon^{2}\right)
$$

which in general does not satisfy the initial condition $\phi\left(z_{0}\right)=\phi_{0}$. The obvious approach to satisfy the boundary condition is to introduce a boundary layer variable $r=\left(z / z_{0}\right)^{1 / \varepsilon}$, $r \geq 1$, as considered by [3]. However, this leads to an unsatisfactory outer expansion 
insofar as the resulting added term to the series is transcendentally large, it being proportional to the homogeneous solution of (11). This causes formal difficulty in the expansion, similar to that in [7], since the algebraic terms in (30) are then relatively exponentially small. A remedy is to subtract a multiple of the homogeneous solution to (11), and to define

$$
\Phi(z)=\phi(z)-A\left(\frac{z}{z_{0}}\right)^{1 / \varepsilon}
$$

so that $\Phi(z)$ satisfies

$$
\Phi(z)-\varepsilon z \frac{d \Phi(z)}{d z}=\frac{1}{z-2}
$$

and the shift $A=\phi_{0}-\Phi\left(z_{0}\right)$ is chosen to enable the asymptotic expansion of $\Phi(z)$ to satisfy the initial condition. The expansion for $\Phi(z)$ is the same as that in (30), namely

$$
\Phi(z)=\frac{1}{z-2}-\frac{\varepsilon z}{(z-2)^{2}}+O\left(\varepsilon^{2}\right)
$$

and thus $A$ is then

$$
A=\phi_{0}-\frac{1}{z_{0}-2}+\frac{\varepsilon z_{0}}{\left(z_{0}-2\right)^{2}}+O\left(\varepsilon^{2}\right),
$$

where (34) is asymptotic to $A$ for small, positive $\varepsilon$. Reference to Sec. 2 indicates that $A=\phi_{0}-I\left(z_{0}\right)$ from (13), $\Phi(z)=I(z)$ from (15), where this comparison requires the expansion of the product term in the denominator of (15) for small, positive $\varepsilon$.

The outer expansion breaks down as $z \rightarrow 2$. An appropriate inner variable is

$$
u=\left(\frac{z}{2}\right)^{1 / \varepsilon}
$$

and then (32) becomes

$$
\Phi(u)-u \frac{d \Phi(u)}{d u}=\frac{1}{2\left[u^{\varepsilon}-1\right]} .
$$

A regular expansion in powers of $\varepsilon$ may be written in the form

$$
\Phi(u)=C(\varepsilon) u+\frac{u}{2 \varepsilon} f_{\ln u}^{\infty} \frac{e^{-r}}{r} d r-\frac{1}{4}+O(\varepsilon),
$$

where $C(\varepsilon)$ is to be determined by matching to the outer expansion. This inner expansion, valid for $u=O(1)$, breaks down when $|\ln u|=O\left(\varepsilon^{-1}\right)$. Therefore, to perform the matching, we introduce an intermediate variable $\eta$ defined by $u=e^{-\eta / \varepsilon}$, with $\varepsilon \ll \eta \ll 1$ in the overlap region. In terms of the intermediate variable, the outer solution (33) takes the following form in the overlap region:

$$
\Phi(\eta)=-\frac{1}{2 \eta}-\frac{1}{4}-\frac{\varepsilon}{2 \eta^{2}}+\cdots
$$

whilst the inner expansion becomes

$$
\Phi(u)=C(\varepsilon) e^{-\eta / \varepsilon}+\frac{e^{-\eta / \varepsilon}}{2 \varepsilon} f_{-\eta / \varepsilon}^{\infty} \frac{e^{-r}}{r} d r-\frac{1}{4}+O(\varepsilon) .
$$

To match (38) and (39), the integral in (39) is expanded for $\varepsilon \ll \eta \ll 1$ by repeated integration by parts. It is then found that the two expansions do match algebraically 
in the overlap region and this can be carried out to all algebraic orders (all powers of $\varepsilon$ ). The constant $C$ is, however, undetermined, since the exponential term in (39) is transcendentally small. This is the same problem which we described in the introduction. It is tempting, but incorrect, to follow [7] and simply put $C=0$, on the basis that there are no corresponding exponential terms in the outer expansion. This is dangerous, since the algebraic outer expansion may conceal exponentially small correction terms that can be evaluated by resummation. We now suggest two ways in which this problem can be surmounted.

3.1. Borel resummation. Suppose that the series

$$
\psi(\varepsilon)=\sum_{n=0}^{\infty} \frac{a_{n} \varepsilon^{n}}{n !}
$$

converges for small $\varepsilon$, and define

$$
B(\varepsilon)=\int_{0}^{L / \varepsilon} e^{-s} \psi(\varepsilon s) d s
$$

where $L>0$ is arbitrary but fixed; then

$$
B \sim \sum_{n=0}^{\infty} a_{n} \varepsilon^{n} \quad \text { as } \varepsilon \rightarrow 0
$$

Since $\psi$ is defined as a series, so also is $B$, and it is the Borel resummation of $\psi$. The proof of this is straightforward, and is Watson's lemma in reverse. The important point is that if a function is only defined by its asymptotic expansion, then we can equivalently define it through the Borel resummation of its asymptotic expansion. Furthermore, the ability to prescribe $L$ enables us to be specific about exponentially small terms in the function.

First we extend Borel resummation to differential operators. If

$$
\psi(\varepsilon D)=\sum_{n=0}^{\infty} \frac{a_{n}(\varepsilon D)^{n}}{n !}
$$

and

$$
B(\varepsilon D)=\int_{0}^{L / \varepsilon} e^{-s} \psi(\varepsilon s D) d s
$$

then

$$
B(\varepsilon D) f(\eta) \sim \sum_{n=0}^{\infty} a_{n}(\varepsilon D)^{n} f(\eta) \quad \text { as } \varepsilon \rightarrow 0,
$$

where $D$ denotes $d / d \eta$.

To apply this in the present case, we use the variable $\eta$ introduced above, i.e.,

$$
z=2 e^{-\eta}
$$

so that (11) is (for $\Phi$ defined by (31))

$$
(1+\varepsilon D) \Phi=\frac{-1}{2\left(1-e^{-\eta}\right)} .
$$


The outer solution is then constructed from the formal inverse

$$
\Phi \sim-\frac{1}{2} \sum_{n=0}^{\infty}(-\varepsilon D)^{n} \frac{1}{\left(1-e^{-\eta}\right)} .
$$

The construction of the general term is, at the least, awkward, but the necessity for doing this will now be bypassed. If we define

$$
\psi(\varepsilon D)=\sum_{n=0}^{\infty} \frac{(-\varepsilon D)^{n}}{n !}=e^{-\varepsilon D},
$$

then Borel resummation implies that

$$
\Phi \sim-\frac{1}{2} \int_{0}^{L / \varepsilon} e^{-s} e^{-\varepsilon s D} d s \frac{1}{1-e^{-\eta}} .
$$

Now $e^{-h D} f(\eta)=f(\eta-h)$, so that

$$
\Phi \sim-\frac{1}{2} f_{0}^{L / \varepsilon} \frac{e^{-s} d s}{1-e^{-(\eta-\varepsilon s)}}
$$

the principal value of the integral is included in the case $L>\eta$, and in fact it is convenient to choose $L=\infty$. Thus

$$
\Phi \sim-\frac{1}{2} f_{0}^{\infty} \frac{e^{-s} d s}{1-e^{-(\eta-\varepsilon s)}}
$$

and

$$
A=\phi_{0}-\Phi\left(\eta_{0}\right)
$$

from (31) where $z_{0}=2 e^{-\eta_{0}}$. Note that the arbitrariness of $L$ is equivalent to the arbitrariness of $H$ in Sec. 2.

If (52) is now expanded for small $\eta$, we obtain the form of the outer solution in the overlap region as

$$
\Phi \sim-\frac{1}{2 \varepsilon} f_{0}^{\infty} \frac{e^{-s} d s}{\eta-\varepsilon s}-\frac{1}{4} \cdots,
$$

which matches directly to (38) (via $w=s-\eta / \varepsilon$ in the integral) provided that we choose $C=0$. Note that if $L$ were chosen to be finite, then we would find $C \neq 0$.

The integral (52) is taken as the outer solution and then the shift $A$ is defined by

$$
A=\phi_{0}+f_{0}^{\infty} \frac{e^{-s} d s}{2-z_{0} e^{\varepsilon s}}
$$

We do not provide an illustration comparing approximate with exact solutions, because they are indistinguishable; in the present case the approximation method reproduces the exact solution as the outer approximation. However, if we had chosen $L$ to be finite, then this would not have been the case. 
3.2. Evaluating exponentially small terms. The above procedure relies on being able to construct the exponentially accurate Borel resummed integral (52); this requires computation, at least in principle, of the general term of the outer solution, which for more complicated differential equations may well not be available. What we require is a practical way of computing exponentially accurate approximations without the general term of the outer (33) and inner (37) expansions. We can do this by applying the resumming procedure in the overlap region. The thinking behind this idea is that the exponential corrective term $S_{N}(z)$ in (23) increases as $z \rightarrow 2$, and is thus maximal in the overlap domain. Below we find by direct computation that this term decreases again in the inner region. Therefore, we can hope that inclusion of this term, computed in the overlap region, actually provides a uniformly accurate corrective term over the whole domain; this assertion appears to be borne out by comparison with numerical results.

In terms of the overlap variable, (32) is

$$
\Phi(\eta)+\varepsilon \frac{d \Phi(\eta)}{d \eta}=\frac{1}{2\left(e^{-\eta}-1\right)} .
$$

We define $\psi$ to be the solution of the singularly forced part of $(56)$, that is,

$$
\psi(\eta)+\varepsilon \frac{d \psi(\eta)}{d \eta}=-\frac{1}{2 \eta}
$$

and then write

$$
\Phi(\eta)=\psi(\eta)+\theta(\eta)+\widetilde{H}(\varepsilon) \exp (-\eta / \varepsilon)
$$

where we can take $\theta(\eta)$ as

$$
\theta(\eta)=-\frac{1}{4}+\frac{1}{4} \sum_{n=1}^{\infty} \sum_{m=0}^{2 n-1} \frac{(-1)^{m} B_{2 n} \varepsilon^{2 n-m-1} \eta^{m}}{n m !} .
$$

The series (59) defines $\theta$ as an analytic function of $\eta$, so that the (divergent) asymptotic expansion for $\psi$,

$$
\psi(\eta) \sim-\frac{1}{2 \eta} \sum_{n=0}^{\infty} \frac{n ! \varepsilon^{n}}{\eta^{n}}
$$

provides the exponential term that we seek. We truncate the expansion (60) after $N$ terms, and write $\psi(\eta)=F(\eta)+R_{N}(\eta)$, where

$$
F(\eta)=-\frac{1}{2 \eta} \sum_{n=0}^{N-1} \frac{n ! \varepsilon^{n}}{\eta^{n}}
$$

is a Poincaré series and the remainder $R_{N}(\eta)$ then satisfies

$$
R_{N}(\eta)+\varepsilon \frac{d R_{N}(\eta)}{d \eta}=-\frac{\varepsilon^{N} N !}{2 \eta^{N+1}}
$$

Following the same procedure as in Sec. 2, we note that the right-hand side of (62) is minimal at

$$
N=\frac{1}{\varepsilon}\left(\eta-\frac{\varepsilon}{2}+O\left(\varepsilon^{2}\right)\right) .
$$


Using this value of $N$ in (62) and expanding for small, positive $\varepsilon$, we obtain to leading order

$$
R_{N}(\eta)+\varepsilon \frac{d R_{N}(\eta)}{d \eta}=-\sqrt{\frac{\pi}{2 \varepsilon \eta}} e^{-\eta / \varepsilon} .
$$

Comparison of (64) to the differential equation for $S_{N}(z)$ from (21), written in terms of $\eta$ and expanded for $\varepsilon \ll \eta \ll 1$, shows that the series for $\psi(\eta)$ does indeed generate the first-order exponentially small correction within the overlap region. Integrating, we find that

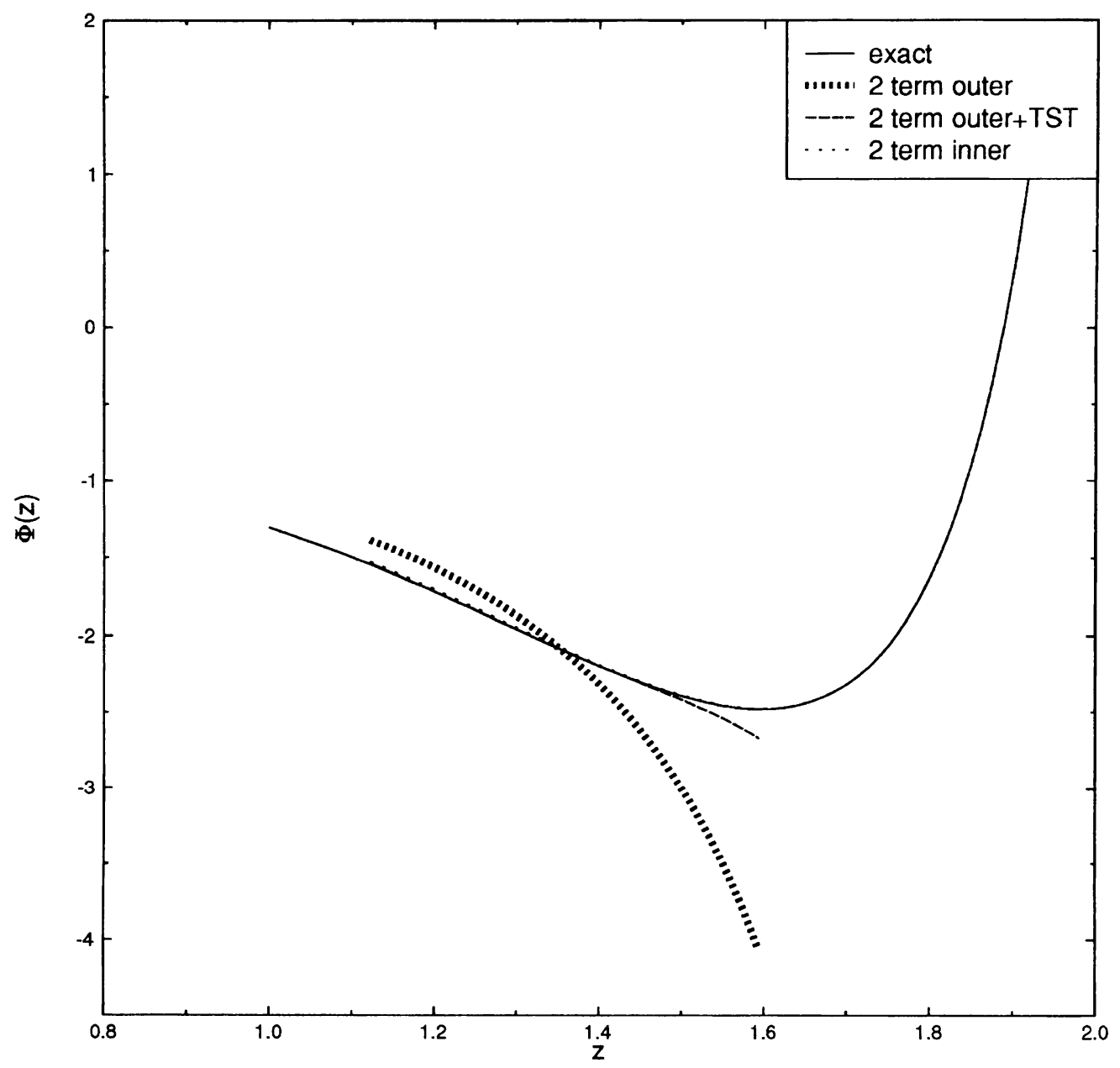

FIG. 3. The numerical solution of the transformed problem in (32) for $\Phi(z)$ is depicted for $\varepsilon=1 / 6$, over the interval $0 \leq z \leq 2$ where the overlap region is approximately $0.7 \ll z \ll 1.7$. Also shown is the two-term outer expansion for $\Phi(z)$ from (33), the two-term inner expansion of $\Phi(u)$ from (37), and the exponentially small corrected overlap solution $\Phi(\eta)$ from (66). The improvement in $\Phi$ within the overlap region is dramatic. $\Phi(u)$ is exponentially accurate as expected. 


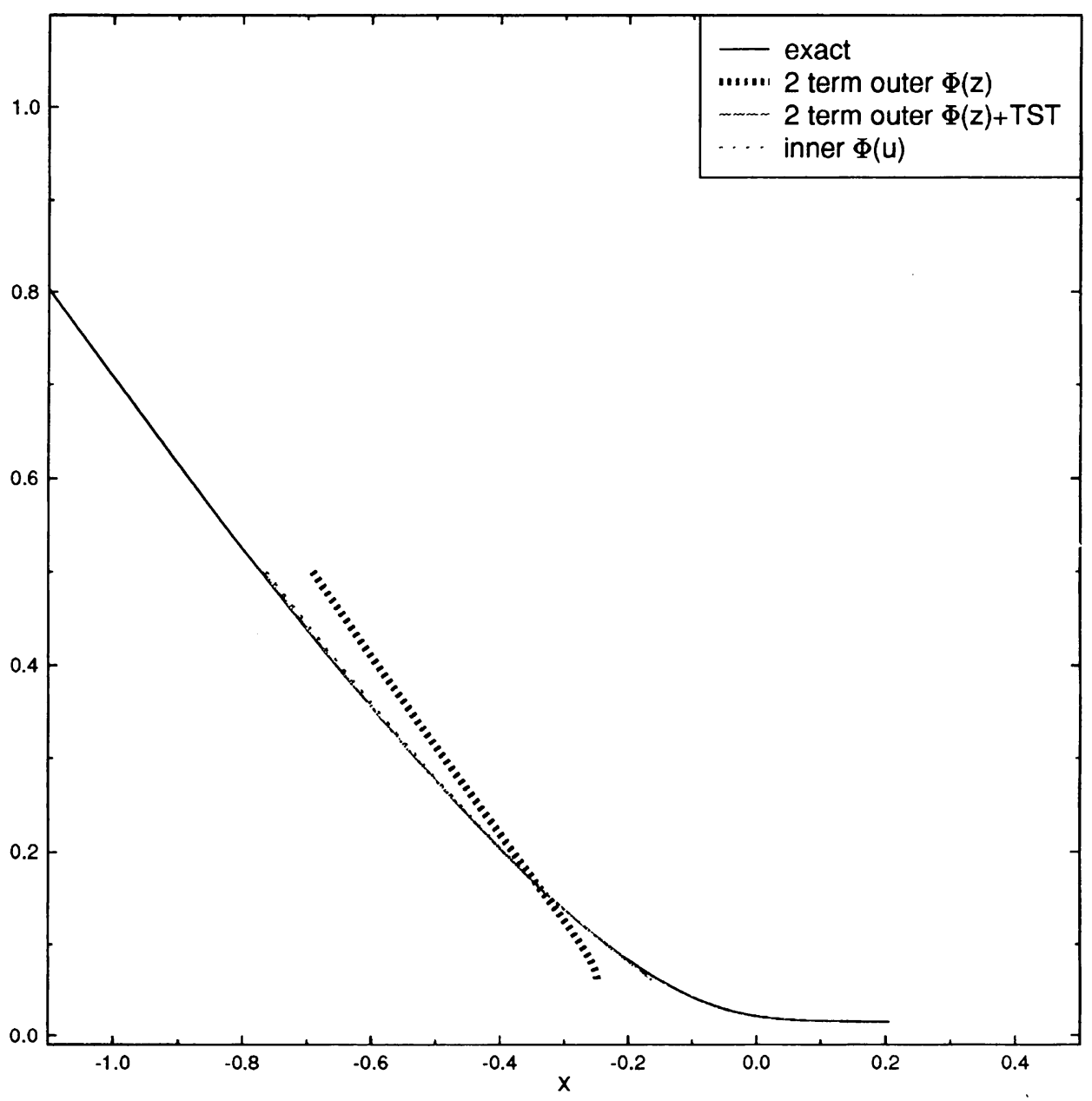

FIG. 4. The results in Fig. 3 are portrayed in terms of the original variables $y$ and $x$ to enable comparison to the results in Fig. 1. Inspection of Figures 1 and 4 indicates that $Y_{2}(\xi)$ is the counterpart of $\Phi(z)$. Thus, $R_{N}(\eta)$ is the numerically significant, exponentially small correction to $Y_{2}(\xi)$ in Sec. 1, and the improvement in $Y_{2}(\xi)$ is dramatic.

$$
R_{N}(\eta)=-\frac{\sqrt{2 \pi \eta}}{\varepsilon^{3 / 2}} e^{-\eta / \varepsilon}
$$

where the constant of integration can be taken as zero, in view of the as yet unspecified constant $\widetilde{H}(\varepsilon)$ in (58) above. Hence, within the overlap region, the solution $\Phi(\eta)$ is taken to be

$$
\Phi(\eta) \sim\left[-\frac{1}{2 \eta}-\frac{1}{4}-\frac{\varepsilon}{2 \eta^{2}}+\cdots\right]-\frac{\sqrt{2 \pi \eta}}{\varepsilon^{3 / 2}} e^{-\eta / \varepsilon}+\widetilde{H}(\varepsilon) e^{-\eta / \varepsilon}
$$


which is composed of a Poincaré series in $\varepsilon$ contained within the square brackets, together with the leading-order exponentially small terms.

Determination of the free constant $\widehat{H}(\varepsilon)$ cannot be done within the scope of these Poincaré expansions. We use a form of numerical matching, by choosing $\widetilde{H}(\varepsilon)$ to be the value that minimizes the mean squared deviation of the difference between the exact (numerically determined) solution $\Phi(\eta)$ and the exponentially corrected Poincaré series of (66), within the overlap region, which is arbitrarily assigned to be the interval $0.37 \lesssim \eta \lesssim 0.4$

In Fig. 3, the two-term expansions of $\Phi(z)(33)$ and $\Phi(u)(37)$ are depicted along with the numerical solution over $0 \leq z \leq 2$ for $\varepsilon=1 / 6$. Using the procedure outlined above, we find that $\widetilde{H}(1 / 6) \approx 0.625 \sqrt{2 \pi} / \varepsilon^{3 / 2}$. The exponentially corrected solution $\Phi(\eta)$ in (66) is also depicted in Fig. 3. The overlap domain is the formal range $1 / 6 \ll \eta \ll 1$, or approximately $0.7 \ll z \ll 1.7$, and in this region $\Phi(\eta)$ represents a dramatic improvement over $\Phi(z)$. In this example, the approximation for $\Phi(u)$ is in fact (and exceptionally) exponentially accurate since the one-term approximation to $\Phi(u)$ is identical to $\psi(\eta)$. To enable a comparison to be made with the original problem in Sec. 1, the results in Fig. 3 are shown in terms of the original variables $y$ and $x$, and these results are shown in Fig. 4. A qualitative inspection of Figures 1 and 4 indicates that $Y_{2}(\xi)$ is the counterpart of $\Phi(z)$, while $y_{3}(\xi)$ (not shown in Fig. 1) is the same for $\Phi(u)$. There is no counterpart for $y_{1}(x)$. The exponentially corrected $\Phi(\eta)$ is a dramatic improvement over $Y_{2}(\xi)$ near the sharp transition.

4. Discussion. The initial motivation in studying this problem was to understand the origin of the sudden switch in solution behaviour at moderate values of $\varepsilon$; limited success in this venture in [3] was tempered by the inability to construct satisfactory asymptotic expansions, because the calculation of matching constants between an inner and an outer solution required solution of the outer problem beyond all algebraic orders of $\varepsilon$. In this paper we have explored this issue, and by comparison with an integral form of the exact solution, we have illuminated two ways in which the required exponentially accurate outer solution can be constructed.

In other problems of this type, it is common to construct exponentially accurate solutions through knowledge of the general asymptotic term in the Poincaré expansion; our desire is to be able to extract this information directly, without explicit knowledge of the general term, and in this paper, we have shown how to do this to a limited extent.

One method can be used where a formal asymptotic expansion can be written in operator-theoretic notation. In that case, the use of Borel summation for operators allows a formally equivalent asymptotic expansion to be written in the form of a Laplace-type integral, which allows explicit prescription of exponentially small terms arising from the integral limits.

Our other method uses the fact that the exponential correction that we seek is maximal in the overlap between inner and outer solutions, where a simplified problem can be solved. This is analogous to Kruskal and Segur's study ([5] and [6]) of the geometric model of crystal growth, where the exponentially small terms are identified as arising through the solution of the differential equation in the vicinity of a pole of the unperturbed 
$(\varepsilon=0)$ solution. In their case, the singularity is complex, whereas for our example, the singularity is on the real axis, and it is this which causes the solution to have an explicit boundary layer structure (in Kruskal and Segur's case, there is an analogous switching region similar to the error function switch that occurs in integral expansions as Stokes's lines are crossed, although Kruskal and Segur were not able to analyse this in their problem).

We have not completely fulfilled our aim of constructing exponentially accurate solutions direct from the differential equation, but we have shown how certain ingredients for doing this are present in our example. In the future it is hoped to extend the insights gained with other similar problems, to elucidate a more general matching procedure which can be used directly.

Acknowledgment. We thank Emmanuele Schiavi for pointing out an error in our original description of Borel summation.

\section{REFERENCES}

[1] M. V. Berry, Uniform asymptotic smoothing of Stokes's discontinuities, Proc. Roy. Soc. London A 422, 7-21 (1989)

[2] R. B. Dingle, Asymptotic Expansions: Their Derivation and Interpretation, Academic Press, New York, 1973

[3] A. C. Fowler, G. Kember, and S. G. B. O'Brien, Small exponent asymptotics, IMA J. Appl. Math., submitted

[4] A. C. Fowler and C. Johnson, Ice sheet surging and ice stream formation, Ann. Glaciol. 23, 68-73 (1996)

[5] M. D. Kruskal and H. Segur, Asymptotics beyond all orders in a model of dendritic crystals, A. R. A. P. Technical Memo, 85-25 (1985)

[6] M. D. Kruskal and H. Segur, Asymptotics beyond all orders in a model of crystal growth, Stud. Appl. Math. 85, 129-181 (1991)

[7] A. D. McGillivray, A method for incorporating transcendentally small terms into the method of matched asymptotic expansions, Studies in Appl. Math. 99(3), 285-310 (1997)

[8] S. B. G. O'Brien, E. G. Gath, A. C. Fowler, and G. Kember, Asymptotics with small exponent in a model for ice-sheet surging, Proc. Roy. Irish Acad., in press

[9] A. B. Olde Daalhuis, S. J. Chapman, J. R. King, J. R. Ockendon, and R. H. Tew, Stokes phenomenon and matched asymptotic expansions, SIAM J. Appl. Math. 55(6), 1469-1483 (1995)

[10] R. B. Paris and A. D. Wood, Stokes phenomenon demystified, IMA Bulletin 31, 1-10 (1995)

[11] H. Segur, S. Tanveer, and H. Levine (eds.), Asymptotics beyond All Orders, NATO Adv. Sci. Inst. Ser. B, Vol. 284, Plenum Press, New York, 1991 